\title{
The Role of Axonopathy in Parkinson's Disease
}

\author{
Karen L. O'Malley* \\ Department of Anatomy and Neurobiology, Washington University School \\ of Medicine, 660 South Euclid Avenue, Saint Louis, Missouri, 63110, USA

\begin{abstract}
New genetic and environmental studies of Parkinson's disease have revealed early problems in synaptic function and connectivity indicating that axonal impairment may be an important hallmark in this disorder. Since many studies suggest that axonal dysfunction precedes cell body loss, it is critical to target axons with treatments aimed at preserving "connectivity" as well as to develop and verify "biomarkers" with which to assess disease progression and drug efficacy.
\end{abstract}

Key words: axon transport, mitochondria, Wallerian degeneration, MPTP

Parkinson's disease (PD) is a common neurodegenerative disorder with at least one million people in the United States and six million worldwide being affected. The clinical symptoms of PD, tremor, rigidity and bradykinesia, primarily arise from the loss of dopaminergic cells in the substantia nigra pars compacta. Characteristic pathological changes include decreased mitochondrial complex I activity and increased indices of oxidative stress as well as the presence of intracellular and axonal deposits called Lewy bodies and Lewy neurites, respectively (Samii et al., 2004). Although the etiology of PD remains unknown, both genetic and environmental factors appear to play a role. A number of genes associated with familial PD have been identified (e.g. a-synuclein, parkin, DJ-1, PINK1, LRRK2, UCHL1, ATP13A2) demonstrating that molecular mechanisms underlying PD are complex and likely to be heterogeneous (Schapira et al., 2009; Westerlund et al., 2010). In addition,

*To whom correspondence should be addressed.

TEL: 314-362-7087, FAX: 314-362-3446

e-mail: omalleyk@wustl.edu

Received December 28, 2010

Accepted for publication December 31, 2010 exposure to toxins such as $\mathrm{N}$-methyl-4-phenyl1,2,3,6-tetrahydropyridine (MPTP) or its active derivative, $\mathrm{MPP}^{+}$, results in a disorder that mimics many aspects of this disease (Westerlund et al., 2010).

Most knowledge about the molecular mechanisms underlying PD has come from postmortem tissue studies as well as the genetic associations and environmental toxins which mimic the disorder (Schapira et al., 2009; Westerlund et al., 2010). In general, these studies support the view that in PD, dopaminergic neurons die via programmed cell death or apoptosis. Thus therapeutic strategies aimed at halting the underlying causes of cell loss in PD have targeted apoptotic pathways with some success, at least in animal models. Unfortunately, there is little clinical evidence to show that blocking cell death is neuroprotective (Waldmeier et al., 2006). These results underscore the need for broadening the models of PD pathogenesis. Furthermore, it is increasingly apparent that preserving critical circuits can only be achieved by "staying connected".

Recently, ultrastructural, physiological, and genetic studies have revealed early abnormalities in 
synaptic function and connectivity in PD. Thus, mounting evidence indicates that axonal dysfunction may be an important hallmark in PD, one that unifies the many disparate models (Hilliard, 2009; Morfini et al., 2009).

How does impaired axonal transport lead to axon pathology? Neurons are the most highly polarized of vertebrate cells with axons extending up to 10,000 times the width of their cell bodies. Thus, neurons depend upon efficient transport systems to get "cargos" (proteins, vesicles, and other organelles) where they need to go. Cargos move along tracks of microtubules with the help of specialized proteins. For instance, cargos are transported in the anterograde direction by kinesin motor proteins while cargos returning to the cell body are largely transported by dynein motors (Cai and Sheng, 2009). When either process is disrupted, cargos can accumulate leading to protein aggregation, the sequestration of cellular factors and organelles, activation of cell stress signaling cascades, disruption of the tracks themselves and ultimately, axonal degeneration. Defective axonal transport will also lead to the loss of new material and/or vesicles at the distal axon, the loss of neurotropic signaling, the failure to degrade defective proteins and potentially the activation of programmed cell death pathways. Finally, even very subtle changes in axon transport may lead to the re-distribution of important organelles such as mitochondria with an ensuing loss of energy and consequently disruption of energy-driven processes such as synaptic func- tion, movement of other cargoes, etc. (Cai and Sheng, 2009). A model of some of these key features is shown in Fig. 1.

What is the evidence that defective axonal transport plays a role in PD? First, there is widespread axonal pathology from the earliest stages of the disorder suggesting that axonal function is impaired (Braak et al., 2004). Moreover, in patient, non-human primate and animal models of PD, the loss of axons appears to precede the loss of cell bodies (Dauer and Przedborski, 2003). For example, the onset of symptoms in patients appears after most of the axonal dopamine in the striatum has been depleted $(70 \sim 80 \%)$ and about half of the nigral dopamine-producing cells have died (Bernheimer et al., 1973). Also, the appearance of $\alpha$-synuclein-positive Lewy neurites almost always precedes that of Lewy Bodies (Braak et al., 2004; Orimo et al., 2008). These observations have led to the hypothesis that nigral neurons degenerate through a "dying back" axonopathy in which degeneration begins in the distal axon and proceeds over weeks or months towards the cell body (Raff et al., 2002).

Second, mutations in the PD-linked Leucine-Rich Repeat Kinase 2 (LRRK2) gene, which represent the most common genetic cause for familial $(6 \%)$ and idiopathic (1 2\%) PD is also associated with aggregated axonal $\alpha$-synuclein as well as with tau pathology (Ballatore et al., 2007). Knockdown of LRRK2 or introduction of "kinase-dead" constructs leads to long, highly branched neuritic processes

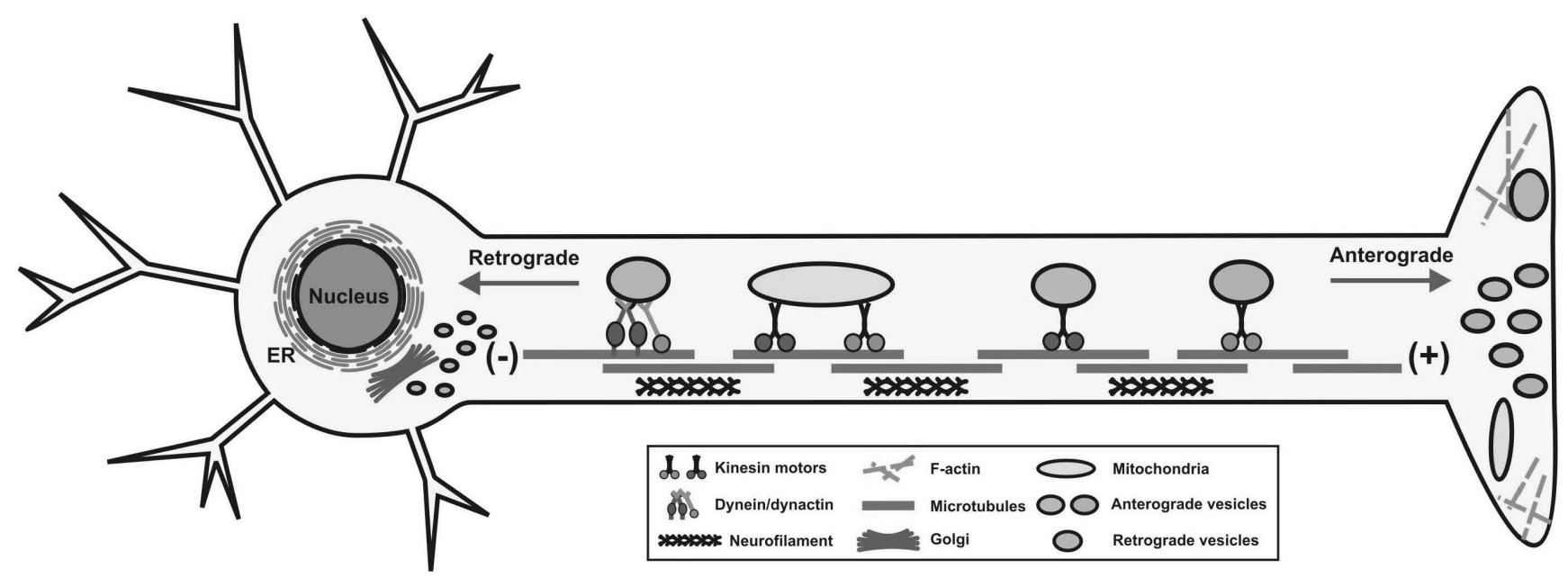

Fig. 1. Key features of axonal transport: cargos, tracks and motor proteins. 
whereas constructs with increased kinase activity exhibit very short simple processes in neuronal cultures or transduced nigrostriatal models (MacLeod et al., 2006). Moreover, transgenic mice expressing the PD-linked mutation LRRK2 (R1441G) recapitulate key features of $P D$ itself including motoric, neurochemical and pathological features ( $\mathrm{Li}$ et al., 2009). Importantly, these animals exhibit an early, progressive decline in dopaminergic terminal fields with a more than 8-fold increase in dystrophic processes and abnormal axonal swellings ( $\mathrm{Li}$ et al., 2009). Thus, these data also support the idea that impaired axonal transport plays an early, pivotal role in LRRK2-associated PD.

Third, $\alpha$-synuclein-positive Lewy neurites precede Lewy bodies in the brain (Braak et al., 2004) as well as in the cardiac sympathetic nervous system (Orimo et al., 2008). Specifically $\alpha$-synuclein aggregates appear first in distal axons then as these regress, aggregates accumulate in more proximal axons and subsequently cell bodies (Braak et al., 2004; Orimo et al., 2008). Conceivably, this early chronology mirrors the pathological mechanism associated with PD. Additionally, $\alpha$-synuclein mutants. accumulate in the cell soma when overexpressed in cortical neurons, suggesting reduced axonal transport as well (Saha et al., 2004). Recent studies examining protein domains associated with transport indicate that those regions involved in membrane binding decreased the number of $\alpha$ -synuclein particles present in hippocampal synapses (Yang et al., 2010). Authors speculate that $\alpha$-synuclein might function as a scaffolding protein linking membranous cargoes with microtubule-dependent axonal transport (Yang et al., 2010)

Fourth, genetic mutations in the PD-linked genes, Parkin, an E3 ligase, and PINK1 (PTEN-induced putative kinase 1 protein) a mitochondrially-targeted kinase, have been shown to regulate mitochondrial fission/fusion machinery and alter axonal transport (Lu, 2009; Bueler, 2010). For example, mitochondria are not only transported up and down the axon they also undergo dynamic interconversion events in which two mitochondria fuse to form a long tubular structure (fusion) or a single mitochondria splits in two (fission). Fusion/fission processes are thought to be intimately involved in mitochondrial transport since 1) mutations associated with Drp1, a fusion promoting gene, impair axonal transport (Lu, 2009); 2) fused, elongated mitochondria are less mobile then short, vesicular structures (Cai and Sheng, 2009); and 3) fission generates healthy mitochondria that are transported down the axon whereas damaged, dysfunctional mitochondria are sent back to the cell body for repair by fusion or degraded via mitophagy (Miller and Sheetz, 2005). Very recent data indicate that PINK 1 is selectively stabilized on depolarized mitochondrial membranes. This in turn acts as a signal for Parkin recruitment which tags Pink-positive, depolarized mitochondria for destruction via autophagy (i.e. mitophagy; Narendra et al., 2010). PINK1 can also form a complex with the key mitochondrial transport proteins, Miro and Milton (Weihofen et al., 2009). Taken together these data suggest that PINK1/ Parkin PD-linked mutations lead to impaired mitochondrial dynamics resulting in altered transport, distribution and loss of synaptic function.

Fifth, environmental toxins known to mimic PD such as rotenone and MPTP or MPP ${ }^{+}$, not only inhibit mitochondrial Complex I activity, but also depolymerize microtubules which take on a beaded, broken appearance leading to axon fragmentation (Cappelletti et al., 2005; Ren et al., 2005). This might be due to a direct interaction or indirectly as a consequence of Complex I inhibition resulting in the loss of ATP and increased oxidative stress (Cappelletti et al., 2005; Ren et al., 2005). In either case, loss of microtubule integrity would clearly lead to axonal degeneration (Fig. 1).

Sixth, recent data suggest that the Wallerian degeneration slow fusion protein $\left(W / d^{S}\right)$ can delay axonal degeneration about 10-fold from a wide variety of genetic and toxin-inducing stimuli in vivo and in vitro. WId ${ }^{S}$ is a chimeric protein composed of the N-terminal 70 amino acids of the ubiquitination factor Ube4b followed by a 18 amino acid linker region and the entire sequence of Nmnat1, a key enzyme in the biosynthesis of $\mathrm{NAD}^{+}$(Mack et al., 2001). Most studies suggest that catalytically active Nmnat1 is necessary for axonal protection although it is unclear whether increased $\mathrm{NAD}^{+}$is responsible (Sasaki et al., 2009). Despite $W / d^{S}$ being primarily localized in the nucleus, subcellular localization studies have found substantial amounts of $W / d^{S}$ within the mitochondrial matrix where it may en- 
hance ATP synthesis (Yahata et al., 2009), prevent free radical formation (Press and Milbrandt, 2009), or maintain mitochondrial membrane potential (lkegami and Koike, 2003). Data from this lab has shown that in the mouse MPTP model, the $W I d^{S}$ gene product enhances survival, prevents nigrostriatal axon degeneration, and attenuates neurotransmitter loss but does not rescue cell bodies (Hasbani and O'Malley, 2006). Despite this striking terminal field preservation, how $W / d^{S}$ prevents axonal dysfunction is unknown but may be related to its effects on mitochondrial function.

Finally results using the isolated squid axoplasm model support the notion that $\mathrm{MPP}^{+}$directly inhibits fast axonal transport independent of mitochondria effects (Morfini et al., 2009). Specifically, MPP ${ }^{+}$ increases retrograde trafficking of organelles in a PKC $\delta$, caspase 3-dependent process. This in turn leads to a loss of appropriate neurotransmission and failure to generate post synaptic responses (Morfini et al., 2009). Taken together, these data provide direct evidence that the PD-mimetic, MPP ${ }^{+}$ rapidly affects critical axonal processes. Moreover, using compartmented chambers and dopaminergic neurons derived from genetically altered animals expressing targeted GFP, we have been able to show that, like the squid giant axon, $\mathrm{MPP}^{+}$affects axonal trafficking (Unpublished results).

Taken together, results from both environmental and genetic factors linked to PD support an early, critical role for axonal impairment in this disorder. Therefore, it is critical to target new drugs to axons in order to preserve "connectivity". At the very least, efforts to delay axonal dysfunction may push back the disease clock allowing those afflicted to maintain an improved quality of life. Ultimately, therapeutics targeted towards both the axon and the cell body may provide the most efficacious treatment of all.

\section{REFERENCES}

Ballatore C, Lee VM and Trojanowski JQ (2007) Tau-mediated neurodegeneration in Alzheimer's disease and related disorders. Nat Rev Neurosci 8:663-672.

Bernheimer $\mathrm{H}$, Birkmayer $\mathrm{W}$, Hornykiewicz $\mathrm{O}$, Jellinger $\mathrm{K}$ and Seitelberger $F$ (1973) Brain dopamine and the syndromes of Parkinson and Huntington. Clinical, morphological and neurochemical correlations. J Neurol Sci 20:415-455.
Braak H, Ghebremedhin E, Rüb U, Bratzke H and Del Tredici K (2004) Stages in the development of Parkinson's disease-related pathology. Cell Tissue Res 318:121-134.

Bueler H (2010) Mitochondrial dynamics, cell death and the pathogenesis of Parkinson's disease. Apoptosis 15:13361353.

Cai Q and Sheng ZH (2009) Mitochondrial transport and docking in axons. Exp Neurol 218:257-267.

Cappelletti G, Surrey T and Maci R (2005) The parkinsonism producing neurotoxin MPP ${ }^{+}$affects microtubule dynamics by acting as a destabilising factor. FEBS Lett 579:47814786.

Dauer W and Przedborski S (2003) Parkinson's disease: mechanisms and models. Neuron 39:889-909.

Hasbani DM and O'Malley KL (2006) WId(S) mice are protected against the Parkinsonian mimetic MPTP. Exp Neurol 202:93-99.

Hilliard MA (2009) Axonal degeneration and regeneration: a mechanistic tug-of-war. J Neurochem 108:23-32.

Ikegami K and Koike T (2003) Non-apoptotic neurite degeneration in apoptotic neuronal death: pivotal role of mitochondrial function in neurites. Neuroscience 122:617-626.

Li Y, Liu W, Oo TF, Wang L, Tang Y, Jackson-Lewis V, Zhou C, Geghman K, Bogdanov M, Przedborski S, Beal MF, Burke RE and Li C (2009) Mutant LRRK2 (R1441G) $B A C$ transgenic mice recapitulate cardinal features of Parkinson's disease. Nat Neurosci 12:826-828.

Lu B (2009) Mitochondrial dynamics and neurodegeneration. Curr Neurol Neurosci Rep 9:212-219.

Mack TG, Reiner M, Beirowski B, Mi W, Emanuelli M, Wagner D, Thomson D, Gillingwater T, Court F, Conforti L, Fernando FS, Tarlton A, Andressen C, Addicks K, Magni G, Ribchester RR, Perry VH and Coleman MP (2001) Wallerian degeneration of injured axons and synapses is delayed by a Ube4b/Nmnat chimeric gene. Nat Neurosci 4:1199-1206.

MacLeod D, Dowman J, Hammond R, Leete T, Inoue $\mathrm{K}$ and Abeliovich A (2006) The familial Parkinsonism gene LRRK2 regulates neurite process morphology. Neuron 52:587-593.

Miller KE and Sheetz MP (2004) Axonal mitochondrial transport and potential are correlated. J Cell Sci 117:27912804.

Morfini GA, Burns M, Binder LI, Kanaan NM, LaPointe N, Bosco DA, Brown RH Jr, Brown H, Tiwari A, Hayward L, Edgar J, Nave KA, Garberrn J, Atagi Y, Song Y, Pigino $G$ and Brady ST (2009) Axonal transport defects in neurodegenerative diseases. J Neurosci 29:12776-12786.

Narendra DP, Jin SM, Tanaka A, Suen DF, Gautier CA, Shen J, Cookson MR and Youle RJ (2010) PINK1 is selectively stabilized on impaired mitochondria to activate Parkin. PLoS Biol 8:e1000298.

Orimo S, Uchihara T, Nakamura A, Mori F, Kakita A, Wakabayashi $\mathrm{K}$ and Takahashi $\mathrm{H}$ (2008) Axonal alpha-synuclein aggregates herald centripetal degeneration of cardiac sympathetic nerve in Parkinson's disease. Brain 131:642-650.

Press C and Milbrandt $\mathrm{J}$ (2008) Nmnat delays axonal degeneration caused by mitochondrial and oxidative stress. $J$ Neurosci 28:4861-4871.

Raff MC, Whitmore AV and Finn JT (2002) Axonal selfdestruction and neurodegeneration. Science 296:868-871. 
Ren $Y$, Liu W, Jiang $H$, Jiang $Q$ and Feng J (2005) Selective vulnerability of dopaminergic neurons to microtubule depolymerization. J Biol Chem 280:34105-34112.

Saha AR, Hill J, Utton MA, Asuni AA, Ackerley S, Grierson AJ, Miller CC, Davies AM, Buchman VL, Anderton BH and Hanger DP (2004) Parkinson's disease alpha-synuclein mutations exhibit defective axonal transport in cultured neurons. J Cell Sci 117:1017-1024.

Samii A, Nutt JG and Ransom BR (2004) Parkinson's disease. Lancet 363:1783-1793.

Sasaki Y, Vohra BP, Lund FE and Milbrandt J (2009) Nicotinamide mononucleotide adenylyl transferase-mediated axonal protection requires enzymatic activity but not increased levels of neuronal nicotinamide adenine dinucleotide. J Neurosci 29:5525-5535.

Schapira AH, Agid Y, Barone $\mathrm{P}$, Jenner $\mathrm{P}$, Lemke MR, Poewe W, Rascol O, Reichmann H and Tolosa E (2009) Perspectives on recent advances in the understanding and treatment of Parkinson's disease. Eur J Neurol 16:10901099.
Waldmeier P, Bozyczko-Coyne D, Williams $M$ and Vaught JL (2006) Recent clinical failures in Parkinson's disease with apoptosis inhibitors underline the need for a paradigm shift in drug discovery for neurodegenerative diseases. Biochem Pharmacol 72:1197-1206.

Weihofen A, Thomas KJ, Ostaszewski BL, Cookson MR and Selkoe DJ (2009) Pink1 forms a multiprotein complex with Miro and Milton, linking Pink1 function to mitochondrial trafficking. Biochemistry 48:2045-2052.

Westerlund M, Hoffer B and Olson L (2010) Parkinson's disease: Exit toxins, enter genetics. Prog Neurobiol 90: 146-156.

Yahata N, Yuasa S and Araki T (2009) Nicotinamide mononucleotide adenylyltransferase expression in mitochondrial matrix delays Wallerian degeneration. J Neurosci 29: 6276-6284.

Yang ML, Hasadsri L, Woods WS and George JM (2010) Dynamic transport and localization of alpha-synuclein in primary hippocampal neurons. Mol Neurodegener 5:9. 